\title{
How to Resolve Conflict in Strategic Alliance: A Cognitive-Map-Based Approach
}

\author{
Tao Zhang and Yanping Liu \\ School of Economics and Management, Beijing Jiaotong University, Beijing100044, P.R. \\ China enmasse@126.com mf001455@263.net
}

\begin{abstract}
With the business environment become more complex and dynamic, organizations have been faced with more pressures of competition. Organizations are often compelled to form strategic alliances with other organizations to survive and prosper. The inter-organizational arrangement can become favorable seedbed for inter-organization conflict. The participants in the strategic alliance often spend a substantial proportion of time and energy to handle conflict situations and realize collaboration. The effectiveness of the efforts depends on their comprehension on the characteristics of conflict, but it is often difficult for relative participants in conflict to understand cognitively conflict situation and manage it efficiently. Cognitive map can represent conceptual causal relationships between different variables, so it can be used to describe the perceptions of participants about the subjective conflict situation. In this paper, we introduce a method based on NPN logic cognitive map to capture and resolve strategic alliance conflicts.
\end{abstract}

Keywords: Strategic alliances, NPN logic, Cognitive map, Conflict resolution

\section{INTRODUCTION}

With the business environment become more complex and dynamic, organizations have been faced with more pressures of competition. Organizations are often compelled to form strategic alliances with other organizations to survive and prosper. The inter-organizational arrangement can bring some advantages to participants in alliance such as access of important resources, knowledge creation, and risk sharing etc, but it can also become favorable seedbed for inter-organization conflict. Moderate conflict is considered necessary for the efficiency and effectiveness of strategic alliance, but intense conflict can produce negative residues, even undermine the basis of strategic alliance. Therefore, conflict management and resolution in strategic alliance is of significance on the success of strategic alliance.

Conflict in strategic alliance is a process in which one partnering organization perceives that its interests are opposed or negatively affected by another partnering organization. The participants in strategic alliance often spend a substantial proportion of time and energy to handle conflict situations and realize collaboration. The effectiveness of the efforts depends on their comprehension on the characteristics of conflict, but it is often difficult for relative participants in conflict to understand cognitively conflict situation and manage it efficiently. Cognitive map (CM) can 
represent conceptual causal relationships between different variables, so it can be used to describe the perceptions of participants about the subjective conflict situation. Through cognitive maps construction, partnering organizations in strategic alliance can identify the cause-effect relationships related to conflict situation, and then they can understand each other better and transform their opinions into collective synergy.

In this paper, we introduce a method based on negative-positive-neutral (NPN) logic cognitive map to capture and resolve strategic alliance conflicts. The remaining sections are organized as follows. We first introduce NPN logic which constructs a logical framework for cognitive map modeling, and the concepts of NPN logic cognitive map. Then we describe a generic method to detect and resolve conflict in strategic alliance. And in the end, we propose the managerial implications and an identification of avenues for future research.

\section{LITERATURE REVIEW}

In this part, we will introduce NPN fuzzy logic which constructs a logical framework for cognitive map modeling, and then present general cognitive map concept and NPN logic cognitive map concept.

In NPN fuzzy logic, a singleton variable can assume any real value in the interval $[-1,1]$, or it can be assumed an ordered NPN value pair $(x, y)$, where $x$ and $y$ assume real values in $[-1,1]$, which indicates a negative strength and a positive strength simultaneously considered. Since each singleton value $x$ can also be represented as a pair $(x, x)$, any NPN logic value can be represented as an ordered pair. Thus the ordered pairs (by $\leq$ ) in $[-1,1] \times[-1,1]$ form a complete representation space for all NPN logic values.

An NPN relation $R$ in $X \times Y$ was defined as a collection of ordered pairs or a subset of $X \times Y$ characterized by a membership function $\mu_{R}$ that associates each pair $\left(x_{i}, y_{i}\right)$ in $X \times Y$ with strength of relationship by using an NPN logic value. An NPN relation $R$ in $X \times X$, where $X=\left\{x_{1}, x_{2} \cdots x_{n}\right\}$, is NPN max-* transitive if, for all $i, j$, and $k, 0<i, j, k \leq n$ we have

$$
\mu_{R}\left(x_{i}, x_{k}\right) \geq \max \left(\mu_{R}\left(x_{i}, x_{j}\right) * \mu_{R}\left(x_{j}, x_{k}\right)\right) .
$$

In (1), the operator * stands for a general conjunction operator that may be any $\mathrm{T}$ norm extended from the interval $[0,1]$ to $[-1,1]$. The max - * composition of two NPN relations $R \subseteq X \times Y$ and $Q \subseteq Y \times Z$, denoted by $R \circ Q$, is defined by

$$
\mu_{R \circ Q}=\max \left(\mu_{R}(x, y) * \mu_{Q}(y, z)\right), x \in X, y \in Y, z \in Z .
$$

The operator max is equivalent to OR. The $n$-fold composition of $R$ is denoted as $R^{n}=R \circ R \circ \cdots \circ R$. A max-* transitive closure of an NPN relation $R$ in $X \times X$ is 
defined as the smallest $\max -*$ NPN transitive relation containing $R$. A theorem proved states: given $X=\left\{x_{1}, x_{2} \cdots x_{n}\right\}$ finite set, the $\max -*$ transitive closure $\bar{R}$ of an NPN relation $R$ in $X \times X$ exists and can be computed as

$$
\bar{R}=R^{1}+\cdots+R^{2 n}=\left(R^{1}+\cdots+R^{n}\right) \circ\left([I]+\left(R^{1}+\cdots+R^{n}\right)\right)[1] .
$$

Cognitive Map is a clear representation of the causal relationships that are perceived to exit among the attributes and/or concepts of a given environment [2]. It comprises nodes that represent the crucial factors most relevant to the decision circumstances, and arcs that indicate different causal relationships among factors. $\mathrm{CM}$ has been widely used for knowledge acquisition and processing in different disciplines and research domains where both the system concepts and relationships are basically complex. When the relationships are numerically characterized, the CM approach is an inference mechanism that allows the complex causal relations among factors to be identified and their impact to be constructed. The causal relationships are often indicated through weighted directed connections presented in two-valued logic or classical fuzzy logic which has internal deficiencies mentioned above in assessing the impact of positive and negative causalities when stimuli are exerted on one or more elements in a CM. In NPN logic CM, all the strengths of the relationships can be normalized to real value pair $(x, y)$ in $[-1,1]$, so both negative and positive effects can be retained and integrated in the decision analysis for further reference.

\section{COGNITIVE-MAP-BASED METHOD FOR CONFLICT RESOLUTION IN STRATEGIC ALLIANCE}

Four processes compose the cognitive-map-based model for conflict resolution in strategic alliance, that is, goal identification, cognitive map composition, cognitive derivation and decision analysis process. In the goal identification process, partnering organizations in strategic alliance confirm the common expected goals of strategic alliance. Based on the uniform goals, each partnering organization constructs the local cognitive map respectively to gather structured information about own present operational situations. Then gathered information is pooled together by integrating assertions and local cognitive maps to form a combined cognitive, which is called a primary cognitive map (PCM). In the cognitive map derivation process, max-* heuristic transitive closure, which can be used to identify the maximum causalities between any two concepts in PCM, is derived as an advanced CM (ACM) in which implications and inconsistencies of PCM are clarified. Based on $\mathrm{ACM}$, two most effective casual paths, which cause, respectively, a positive and a negative maximum effect, can be confirmed. In decision analysis process, several strategic alliance conflicts are identified based on the computed causal impact paths and values and some approaches for conflict intervention and resolution can be presented.

Strategic agreement on the overall goals of the alliance is a strong foundation for a supportive overall collaborative environment. Goals structured in strategic alliances determine that partnering organizations interact and their interaction pattern 
determines outcomes. When common goals identified, partnering organizations can develop the cognition of cooperative interdependence which will contribute to longterm relationships. In goal identification process, common goals descriptions are determined in strategic alliance. While common goals have been identified, partnering organization in strategic alliances has individual and diverse goals which are often in conflict with the goals of other partnering organizations. Conflict in strategic alliances can be described as the inter-organizational behavior that occurs when one partnering organization perceives that other partnering organizations counteract its goal achievement and expectation. There are many techniques which can be used to determine and validate the common goals descriptions of strategic alliance such as brainstorm, interview, and document analysis. In this process, 2-3 concepts representing common goals are determined.

Cognitive map construction can be regarded as a knowledge pooling process [1]. In this process, different managers' schema or cognitive structures, which reflect their mental understanding of a particular domain, is integrated to form a combined PCM. The importance of knowledge pooling lies in the fact that no one has perfect and complete knowledge about a large and distributed environment [2]. The construction of PCM includes several phases as follows. First, the appropriate sample size is determined, and then initial structured interview with managers at different levels in partnering organizations is conducted. Second, causal concepts are identified and clustered, and adjacency matrices are completed based on pairwise comparison or structural equation modeling technique. Third, individual cognitive maps are augmented and integrated together to form local cognitive maps of partnering organizations based on the identical causal concepts between individual cognitive maps. At last, global PCM based on the identical causal concepts between local cognitive maps is generated. In cognitive map composition process, both positive and negative assertions are weighted and kept separately to form an NPN compound value. Because both positive and negative effects are important in decision analysis, they should not be summed together if they are not counteractive to each other at the same time or they are not caused by the same path.

In the cognitive map derivation process, max-* heuristic transitive closure is derived as ACM in which implications and inconsistencies of PCM are clarified. In this process, two algorithms suggested in [1] can be adopted, that is, a heuristic transitive closure (HTC) algorithm and a heuristic path searching (HPS) algorithm. The HTC algorithm implements logic arithmetic mentioned above, which computes the heuristic transitive closures of NPN relations. The HPS algorithm confirms two most effective casual paths, which cause a positive and a negative maximum ripple causalities effect. If the positive path is desirable, the negative path will cause the maximum side effect, and vice versa. HTC and HPS algorithm enhance the coherence in PCM and clear up implications among PCM node connections. In PCM, there are many unrelated object pairs and many pairs which are related only by either a negative or positive edge. The HTC algorithm completes the PCM via transitivity, and the HPS algorithm elicits the new relationship between object pairs. Then the pieces of partial knowledge in the PCM are integrated and the new relationships are derived based on overall coherence and completeness in the max-* transitive sense. 
In decision analysis process, The elements of $\bar{R}$ is regarded as a more or less steady state which identifies network expectations on the effects caused by stimuli, and then can be used to guide a dynamic decision process until one or more goals are reached. Through this way, several conflicts between partnering organizations can be identified based on the computed causal impact paths and values. Furthermore, the maximum causality factors in a closure, which present the conflict situations between partnering organizations, can be used as the criteria for finding the most effective paths corresponding to some potential solutions. The major advantage in using NPN relations lies in the fact that both positive and negative causalities are retained in the model. The integrated representation can be used to support multi-criteria decision analysis and the reason about the potential consequences of an action by a partnering organization. Based on the representation, alternative solutions to conflict situations can be arranged in the order as follow: the most effective ones with least side effects, the least effective ones with least side effects, the least effective ones with the most side effects and the ones with both maximum effects and side effects [2].

\section{CONCLUSIONS AND IMPLICATION}

This article proposed a cognitive map method to analyze the conflict in strategic alliance based on the NPN logic which can represent both positive and negative causalities simultaneously. The method comprises four processes for conflict resolution in strategic alliance, that is, goal identification, cognitive map composition, cognitive derivation and decision analysis process.

There are some implications in this method for the practitioners and researchers who interest in conflict resolution in strategic alliance. Primarily, the method can help to facilitate the identification of the causal interactions in partnering organizations in strategic alliance. Second, the method can eliminate the ambiguity in partnering organizations on the sources of conflict and the ways of resolutions. However, there are still several limitations in the method. Whereas this article suggests a pairwisecomparison-based technique and/or structural equation modeling technique in causal values confirmation, the appropriateness of these approaches still remains to be further examined. In addition, the method does not consider the cause-effect relationships with time dimension between interrelated causal concepts. Hence, one of the future directions of this research is to represent the time dimension in the interrelated causal concepts.

\section{REFERENCES}

1. W. Zhang, S. Chen, W. Wang, and R. S. King, A cognitive-map-based approach to the coordination of distributed cooperative agents, IEEE Transaction on System, Man, and Cybernetics. Volume 22, Number 1, pp.103-114, (1992).

2. W. Zhang, S. Chen, and J. C. Bezdek, POOL2: a generic system for cognitive map development and decision analysis, IEEE Transaction on System, Man, and Cybernetics. Volume 19, Number 1, pp.31-39, (1989). 